\title{
Siglec1 suppresses antiviral innate immune response by inducing TBK1 degradation via the ubiquitin ligase TRIM27
}

\author{
Qingliang Zheng ${ }^{1, *}$, Jin $\mathrm{Hou}^{2, *}$, Ye Zhou ${ }^{2}$, Yingyun $\mathrm{Yang}^{3}$, Bing Xie ${ }^{1}$, Xuetao Cao ${ }^{1,2,3}$ \\ ${ }^{I}$ Institute of Immunology, Zhejiang University School of Medicine, Hangzhou, Zhejiang 310058, China $;{ }^{2}$ National Key Laboratory \\ of Medical Immunology \& Institute of Immunology, Second Military Medical University, Shanghai 200433, China; ${ }^{3}$ National Key \\ Laboratory of Medical Molecular Biology \& Department of Immunology, Institute of Basic Medical Sciences, Chinese Academy of \\ Medical Sciences, Beijing 100005, China
}

Type I interferon (IFN) production plays pivotal roles in host antiviral innate immune responses, but an excessive production of type I IFN leads to the development of immunopathological conditions. Investigations on the regulatory mechanisms underlying host type I IFN production are currently of great interest. Here, we found that the expression of lectin family member Siglec1 was upregulated by viral infection in macrophages, which was dependent on the IFN/JAK/STAT1 signaling pathway. Siglec1 was found to negatively regulate viral infection-triggered type I IFN production. Mechanistically, Siglec1 associates with DAP12 to recruit and activate the scaffolding function of SHP2; SHP2 then recruits E3 ubiquitin ligase TRIM27, which induces TBK1 degradation via K48-linked ubiquitination at Lys251 and Lys372. Therefore, viral infection-induced upregulation of Siglec1 feedback loop inhibits type I IFN production and suppresses antiviral innate immune responses. Our study outlines a novel mechanism of negative regulation of type I IFN production, which may help virus to escape immune elimination.

Keywords: Siglec1; innate immune response; type I interferon; TBK1; TRIM27

Cell Research (2015) 25:1121-1136. doi:10.1038/cr.2015.108; published online 11 September 2015

\section{Introduction}

Effective recognition of the invading virus by the host innate immune system is essential for the subsequent triggering of antiviral innate and adaptive immune responses. Upon recognizing viral components, host innate immune cells are activated to produce type I interferon (IFN) and proinflammatory cytokines, which are important for elimination of the invading pathogens $[1,2]$. In particular, the inducible production of type I IFN plays pivotal roles in antiviral immune responses because an appropriate amount of type I IFN induces cellular resis-

*These two authors contributed equally to this work.

Correspondence: Xuetao Cao

Tel: +86-571-8820 8283; Fax: +86-571-8820 8022

E-mail: caoxt@immunol.org

Received 9 March 2015; revised 14 June 2015; accepted 20 July 2015; published online 11 September 2015 tance to viral infection and triggers apoptosis of virus-infected cells [3]. However, excessive production of type I IFN may lead to tissue damage, contributing to the development of immunopathological conditions or immune disorders such as autoimmune diseases. Hence, type I IFN production during viral infection should be tightly controlled in order to initiate an appropriate immune response that eliminates invading pathogens while avoiding the development of immune disorders [4]. However, the mechanisms underlying the precise control of type I IFN production and function remain to be fully understood.

Viruses are recognized by the host through pattern recognition receptors (PRRs), including Toll-like receptors, RIG-I-like receptors, Nod-like receptors (NLRs), and nucleic acid sensors $[5,6]$. Upon viral infection, these PRRs trigger TBK1 activation through the key adaptors TRIF, MAVS, or STING, which then activates the transcription factor IRF3 to induce type I IFN production 
[7]. Various types of PRRs tightly cross-regulate type I IFN production to ensure that an appropriate amount is produced. Indeed, several negative regulators of PRR signaling can function to suppress type I IFN production, which may prevent immune disorders caused by uncontrolled or excessive type I IFN production. For example, NLR family CARD domain containing 5 (NLRC5) [8] and NACHT, LRR, and PYD domains-containing protein 4 (NLRP4) [9] have been identified as negative regulators of PRR signaling. As another layer of regulation, PRR signaling is also cross-modulated by signals generated via other membrane-associated receptors. For example, sialic acid binding immunoglobulin-like lectin $\mathrm{G}$ (Siglec-G) negatively regulates type I IFN production in the innate immune response against RNA virus infection by promoting RIG-I proteasomal degradation [10]. However, whether antiviral innate immune response is regulated by other membrane-associated receptors, especially other Siglec family members, still needs investigation.

Siglec1, the first Siglec family member identified, contains 17 immunoglobulin-like domains with the sialic acid-binding site located within the V-set terminal immunoglobulin domain. Siglec1 lacks inhibitory cytosolic signaling motifs and possesses a neutral transmembrane domain [11]. Siglec1 has been shown to play an important role in the capture and internalization of pathogens via binding to sialic acid residues expressed on the surface of some important pathogens such as HIV-1 [12] and porcine reproductive and respiratory syndrome virus [13]. Siglec1 has also been shown to participate in antigen presentation and induction of adaptive immune responses $[14,15]$. However, the biological functions of Siglec1 in antiviral innate immunity remain elusive, and its cytosolic signaling pathway is still unknown.

Ubiquitination is one of the most versatile post-translational modifications (PTMs) of proteins and plays critical roles in the regulation of antiviral innate immune response. Modification of TBK1 by different types of ubiquitin chains has been found to be a key event in the regulation of TBK1-triggered IRF3 activation [16]. K63linked ubiquitination by E3 ubiquitin ligases TRAF3 [17], mind bomb 1 and 2 (MIB1 and MIB2) [18], and Nrdp1 [19] has been shown to induce TBK1 activation. On the other hand, K48-linked ubiquitination of TBK1 can promote the proteasomal degradation of TBK 1 and then terminate the downstream signaling. For instance, NLRP4 recruits the E3 ligase DTX4 to TBK1 for K48-linked polyubiquitination at Lys670, which leads to TBK1 degradation [9]. TRAF-interacting protein (TRIP) also negatively regulates antiviral innate immune response by promoting proteasomal degradation of TBK1 [20]. However, whether other E3 ubiquitin ligases contribute to TBK1 degradation still needs to be fully elucidated.

In the present study, we found that viral infection significantly upregulated Siglec1 expression in mouse macrophages in an IFN/JAK/STAT1 pathway-dependent manner. This viral infection-induced upregulation of Siglec1 facilitated viral replication by suppression of type I IFN production via promoting TRIM27-mediated TBK1 degradation. Our findings reveal a new negative regulatory mechanism for type I IFN production in the antiviral innate immune response.

\section{Results}

Viral infection significantly upregulates Siglec1 expression in macrophages via the IFN/JAK/STAT1 pathway

We previously reported that several genes of the lectin family were significantly upregulated upon VSV infection in macrophages, and siglec1 was one of them [10]. However, the biological significance of Siglec1 upregulation in the antiviral innate immune response still needs to be elucidated. Here we further examined Siglec1 expression in infected mouse peritoneal macrophages and observed that the upregulation of Siglec1 expression following VSV infection occurred in a time- and dose-dependent manner (Figure 1A). Infection of SeV (another RNA virus) or HSV (a DNA virus) also induced Siglec1 upregulation in macrophages (Figure 1B and 1D). In addition to its upregulation in macrophages, Siglec1 expression was also enhanced by VSV infection in mouse peripheral blood mononuclear cells, myeloid conventional dendritic cells (cDCs) and plasmacytoid dendritic cells (Figure 1C). Taken together, these data suggest that infection with various viruses can significantly upregulate Siglec1 expression in innate immune cells.

The mechanism underlying Siglec1 upregulation was further examined. We first determined whether Siglec1 was upregulated by signaling events upstream of IFN production or downstream of the IFN/JAK/STAT1 effector signaling pathway. We found that VSV infection or IFN- $\alpha$ treatment markedly upregulated Siglec1 expression in wild-type (WT) macrophages, and Siglec1 induction was abolished in type I IFN receptor (IFNAR)-deficient macrophages (Figure 1E), suggesting that Siglec1 upregulation is dependent on signals downstream of type I IFN production. We also found an IFN-stimulated response element (ISRE) site located 3048 bp upstream of the transcription start site of Siglec1 (Supplementary information, Figure S1A). We constructed luciferase reporters driven by the Siglec1 promoter containing either WT or mutant ISRE site, and observed that the mutant Siglec1 reporter failed to be induced by IFN- $\alpha$ stimulation (Supplementary information, Figure S1B). 

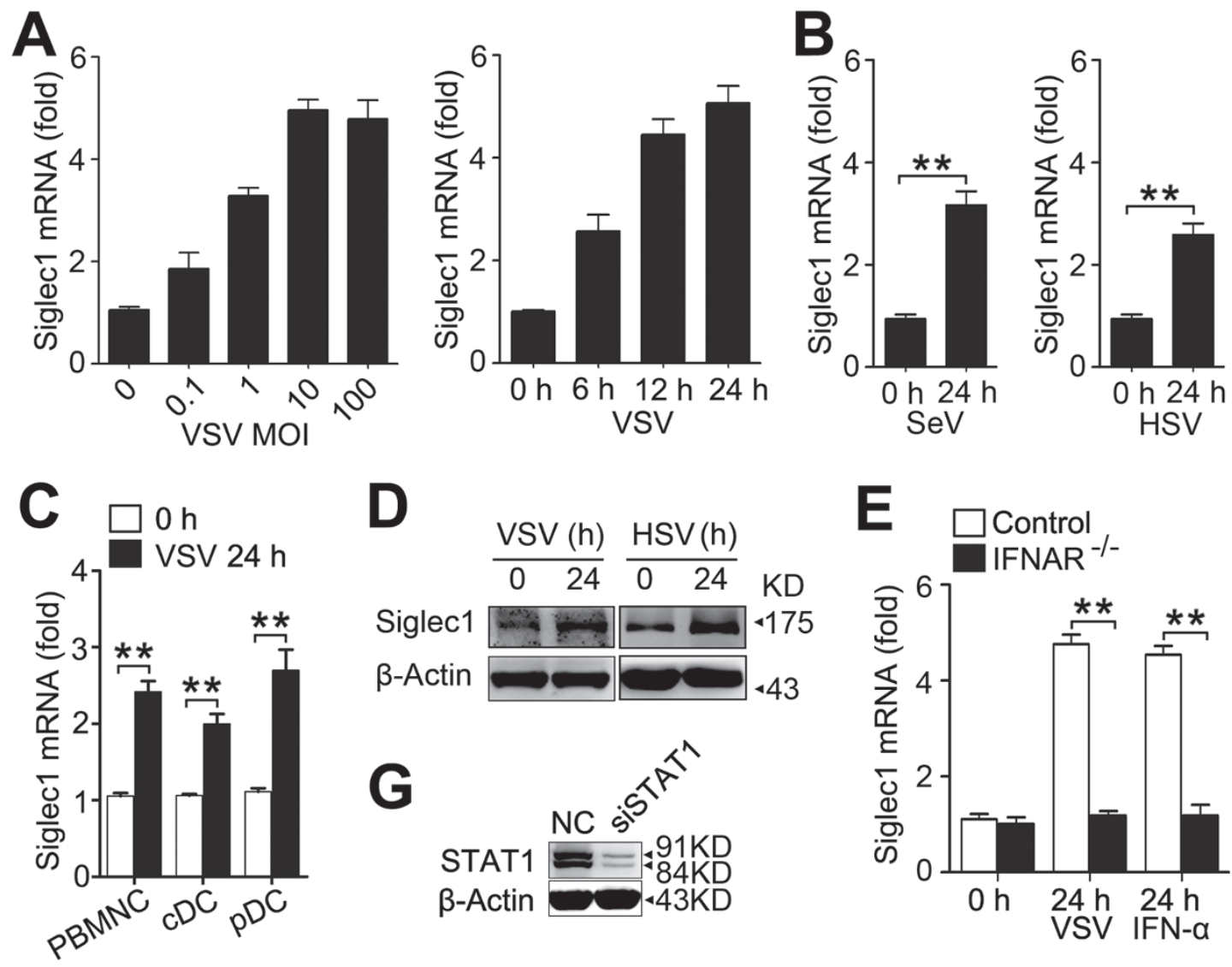

D
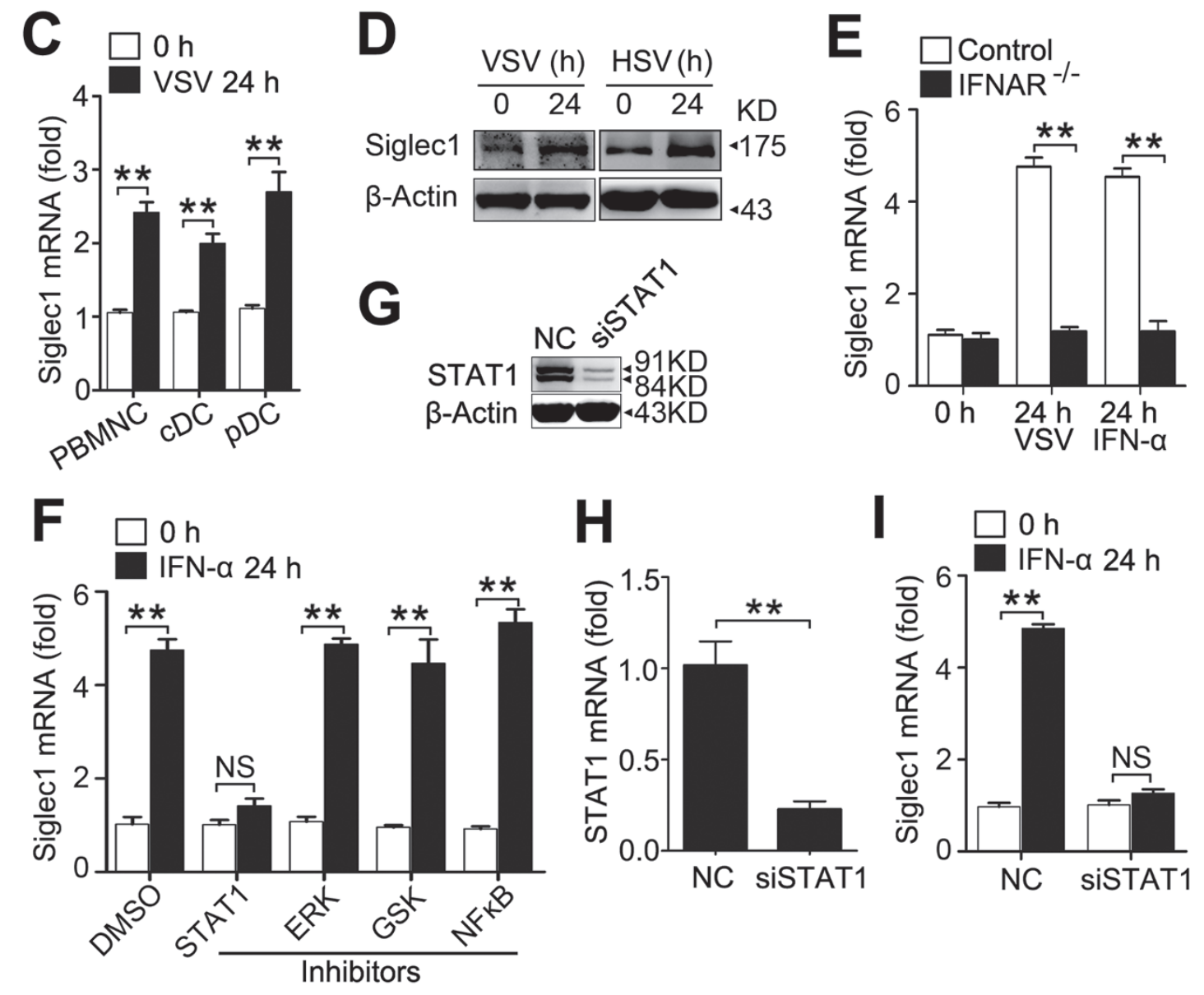

Figure 1 Viral infection upregulates Siglec1 expression through the IFN/JAK/STAT1 signaling pathway in macrophages. (A) Q-PCR analysis of Siglec1 in primary peritoneal macrophages infected with VSV for $24 \mathrm{~h}$ (left), or infected with VSV (MOI = 10) for the indicated time (right). Siglec1 expression was normalized to that of the $\beta$-actin internal control in each sample. (B) Q-PCR analysis of Siglec1 in macrophages infected with SeV $(\mathrm{MOI}=10)$ or HSV $(\mathrm{MOI}=10)$ for $24 \mathrm{~h}$ as indicated. $(\mathrm{C})$ Q-PCR analysis of Siglec1 in peripheral blood mononuclear cells (PBMNCs), cDCs and plasmacytoid dendritic cells (pDCs) infected with VSV $(\mathrm{MOI}=10)$ for $24 \mathrm{~h}$ as indicated. (D) Immunoblot analysis of Siglec1 in macrophages infected with VSV $(\mathrm{MOI}=10)$ or HSV $(\mathrm{MOI}=10)$ for $24 \mathrm{~h}$. $\beta$-Actin served as a loading control. (E) Q-PCR analysis of Siglec1 expression in macrophages from WT or IFNAR-deficient mice treated with VSV $(\mathrm{MOI}=10)$ or IFN- $\alpha(200 \mathrm{U} / \mathrm{ml})$. (F) Q-PCR analysis of Siglec1 in macrophages pretreated for $30 \mathrm{~min}$ with the indicated inhibitors and stimulated with $200 \mathrm{U} / \mathrm{ml} \mathrm{IFN- \alpha} \mathrm{for} 24 \mathrm{~h}$. (G, H) Immunoblot and Q-PCR analyses of STAT1 in macrophages transfected with STAT1 siRNA. (I) Q-PCR analysis of Siglec1 in macrophages transfected with STAT1 siRNA and then stimulated with IFN- $\alpha(200 \mathrm{U} / \mathrm{ml})$ for $24 \mathrm{~h}$. Data are shown as mean \pm SD. ${ }^{* *} P<0.01$; NS, not significant. See also Supplementary information, Figure S1. 
Moreover, inhibition of STAT1 efficiently blocked IFN$\alpha$-induced Siglec1 upregulation, but inhibition of ERK, GSK, or NF- $\kappa$ B activity had little effect (Figure 1F). Consistently, STAT1 knockdown blocked IFN- $\alpha$-induced upregulation of Siglec1 expression (Figure 1G-1I). Taken together, these results suggest that viral infection upregulates Siglec1 expression in macrophages mainly through the IFN/JAK/STAT1 signaling pathway.

Siglec1 promotes VSV replication through inhibiting virus-triggered type I IFN production in macrophages

To investigate the biological significance of VSV-induced upregulation of Siglec1, we examined the effect of Siglec1 knockdown on VSV replication in macrophages. First, we designed two siRNAs targeting Siglec1 (siRNA\#1 and \#2) and determined their knockdown efficiency (Figure 2A). Both irrelevant (NC) and recently reported $\mathrm{C} 911$ seed-matched negative-control siRNA were used to exclude the potential off-target effects of RNA interference [21] (Figure 2A). By measuring the VSV RNA level in the cytoplasm and the $50 \%$ tissue culture infective dose $\left(\mathrm{TCID}_{50}\right)$ per milliliter of the supernatant from the infected macrophages, we found that Siglec1 knockdown suppressed VSV replication (Figure 2B-2C). Moreover, we constructed RAW264.7 macrophage cell clones stably overexpressing Siglec1 (Figure 2D), and found that overexpression of Siglec1 facilitated VSV replication (Figure 2E).

To further determine the role of Siglec 1 in host antiviral responses, we examined the effect of Siglec1 depletion on VSV-triggered type I IFN production. Siglec1 knockdown significantly increased virus-triggered type I IFN production in macrophages and cDCs (Figure $2 \mathrm{~F}-2 \mathrm{G}$ and Supplementary information, Figure S2). Moreover, we found that viral infection-induced type I IFN production was inhibited in macrophages stably overexpressing Siglec1 (Figure 2H), further confirming that Siglec1 could inhibit type I IFN production. These results suggest that upregulation of Siglec1 allows VSV to replicate more effectively in host cells by inhibiting type I IFN production.

Siglec1 inhibits IRF3 phosphorylation by targeting TBKI

We next sought to determine the molecular mechanisms by which Siglec1 inhibits virus-triggered type I IFN production. We found that overexpression of Siglec1 markedly suppressed RIG-I-, MAVS-, or TBK1-induced IFN- $\beta$ luciferase activity. However, Siglec 1 did not affect IFN- $\beta$ luciferase activity induced by the constitutively active IRF3(5D) [22] or IKKe (Figure 3A and Supplementary information, Figure S3). These results suggest that inhibition of type I IFN production by Si- glec1 is dependent on TBK1 and Siglec1 acts upstream of IRF3. VSV infection-triggered phosphorylation of IRF3 and TBK1 was enhanced by Siglec1 knockdown, while phosphorylation of ERK, p38, JNK, and p65 was less influenced (Figure 3B). Moreover, knockdown of Siglec1 resulted in a much higher level of endogenous TBK1 compared with that in control macrophages after poly(I:C) transfection (Figure 3C). Also, overexpression of Siglec1 in macrophages decreased the endogenous TBK1 protein level with little effect on its mRNA level (Figure 3D). Taken together, these results suggest that Siglec1 negatively regulates VSV infection-induced IRF3 activation by reducing TBK1 protein level.

\section{Siglec1 associates with DAP12 and SHP2}

Next, we investigated the molecular mechanisms by which Siglec1 regulates TBK1 protein levels. Since Siglec1 itself lacks inhibitory cytosolic signaling motifs and possesses a neutral transmembrane domain, we sought to identify the adaptor that associates with Siglec1 to mediate the signals that ultimately inhibit type I IFN production. We immunoprecipitated Siglec1 from lysates of VSV-infected peritoneal macrophages and then performed mass spectrometry (MS) to identify Siglec1-associated proteins. Among the proteins identified by the MS, we focused on the adaptor protein DAP12 (containing a cytosolic immunoreceptor tyrosine-based activation motif (ITAM) ) and examined its potential role in regulating $\mathrm{Si}-$ glec1 signaling (Supplementary information, Figure S4).

Some Siglec family members contain a positively charged residue in the transmembrane anchor region, and they may associate with a disulfide bond-linked homodimer of DAP12, DAP10 (containing a cytosolic YxxM motif), or FcR $\gamma$ (containing a cytosolic ITAM motif) [23]. Immunoprecipitation (IP) experiments showed that Siglec1 interacted with ITAM-containing DAP12 and FcR $\gamma$ adaptors, but not DAP10 (Figure 4A). We also observed that the interaction between endogenous Siglec1 and DAP12 was enhanced upon VSV or HSV infection (Figure 4B), suggesting that DAP12 is an important adaptor in Siglec1 signaling pathway.

Considering that DAP12 homodimers contain ITAMs that can be phosphorylated by Src family tyrosine kinases and recruit Syk, SHP-1, or SHP-2 to trigger downstream signaling events [24], we investigated the activation status of these molecules in Siglecl knockdown macrophages in the presence of viral infection. Siglec1 knockdown significantly inhibited SHP2 phosphorylation and activation, while phosphorylation of Syk, Src, and SHP1 was less influenced (Figure 4C), suggesting that Siglec1 is required for VSV-triggered SHP2 phosphorylation. IP experiments also showed that SHP2 interacted 


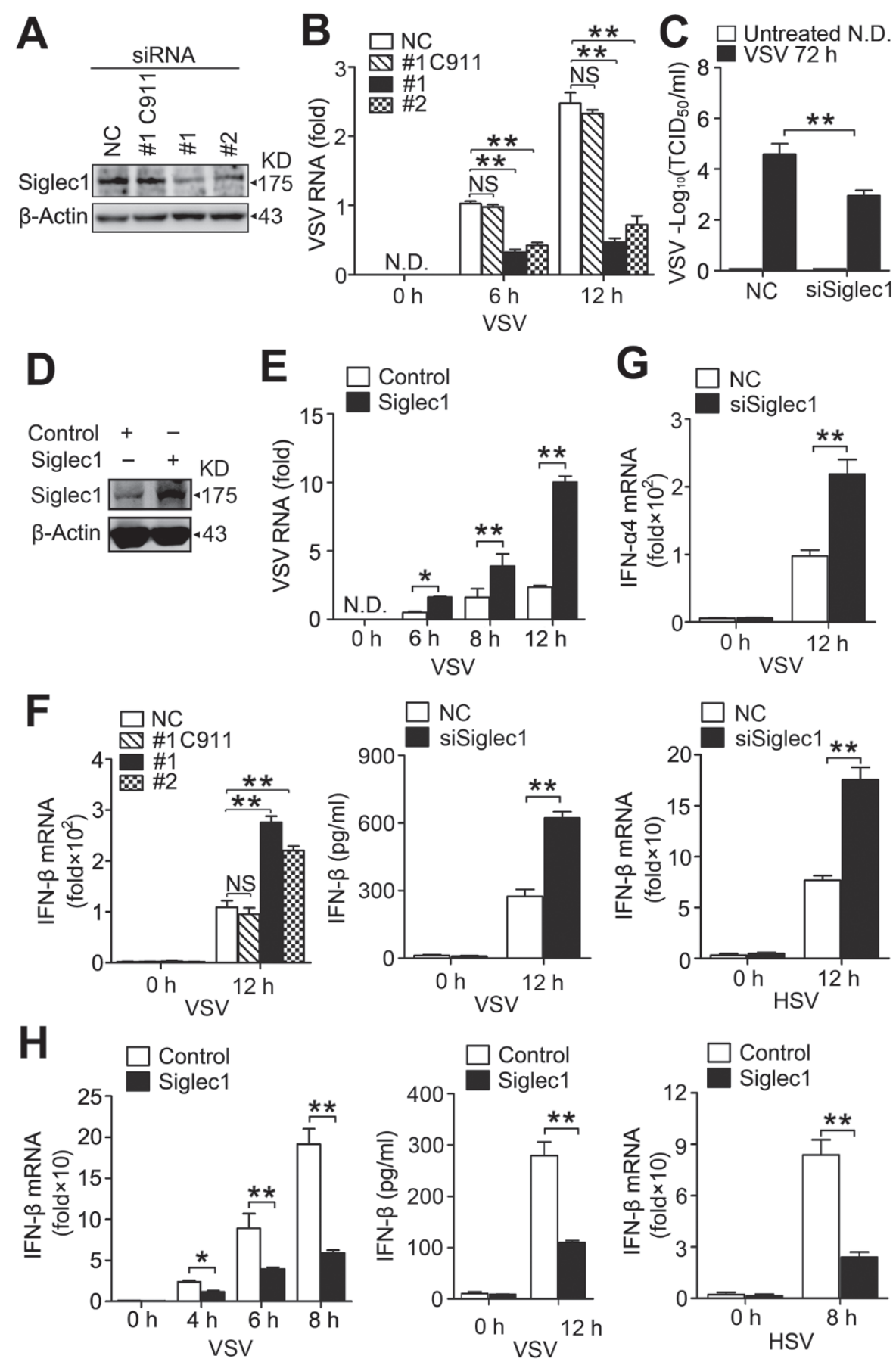

Figure 2 Siglec1 promotes VSV replication and suppresses VSV-triggered type I IFN production. (A) Immunoblot analysis of Siglec1 in macrophages transfected with NC, Siglec1 siRNA\#1 C911, Siglec1 siRNA\#1, and Siglec1 siRNA\#2 as indicated for $48 \mathrm{~h}$. (B) Q-PCR analysis of intracellular VSV RNA levels from macrophages transfected as described in A and stimulated with VSV $(\mathrm{MOI}=10)$ for the indicated time. (C) Macrophages were transfected with Siglec1 siRNA\#1 and infected by VSV $(\mathrm{MOI}=10)$ for $72 \mathrm{~h}$. Their supernatants were measured for VSV TCID ${ }_{50}$. (D) Immunoblot analysis of Siglec1 in RAW264.7 cell clones stably overexpressing Siglec1. (E) Q-PCR analysis of intracellular VSV RNA levels in cells shown in D infected with VSV for the indicated time. (F, G) Macrophages were transfected as in A or C and infected with VSV $(\mathrm{MOI}=10)$ or HSV $(\mathrm{MOI}$ $=10$ ) for $12 \mathrm{~h}$. Q-PCR analysis of IFN- $\beta$ and IFN- $\alpha 4$ mRNA and ELISA of IFN- $\beta$ in the supernatants were then performed. $(\mathrm{H})$ RAW264.7 cell clones stably overexpressing Siglec1 were infected with VSV $(\mathrm{MOI}=10)$ or $\mathrm{HSV}(\mathrm{MOI}=10)$ for the indicated time. Q-PCR analysis of intracellular IFN- $\beta$ mRNA levels and ELISA of IFN- $\beta$ in the supernatants were then performed. Data are shown as mean \pm SD or representative photographs. ${ }^{*} P<0.05$; ${ }^{* *} P<0.01$; NS, not significant. See also Supplementary information, Figure S2. 

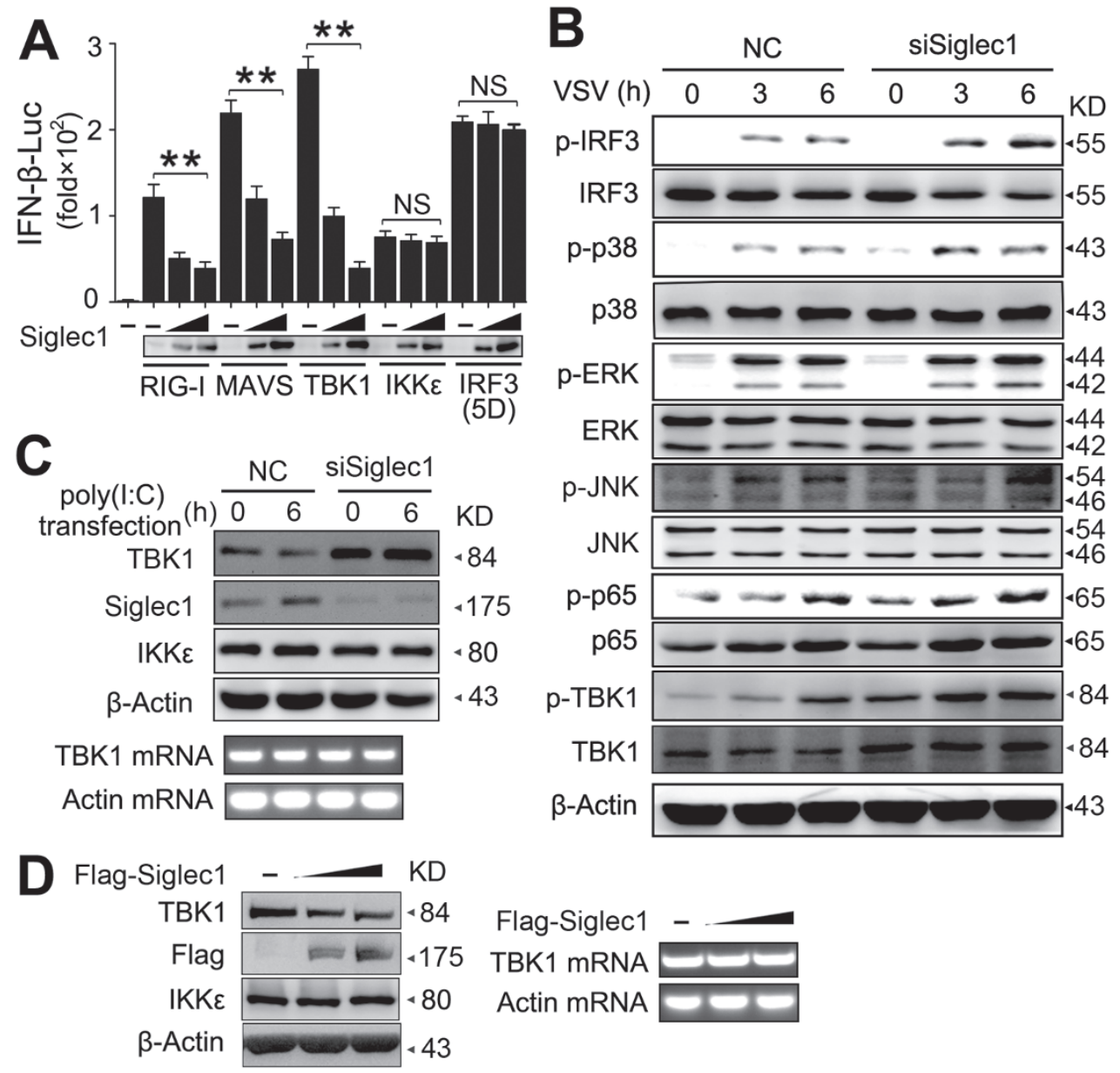

Figure 3 Siglec1 inhibits IRF3 phosphorylation by targeting TBK1. (A) IFN- $\beta$ luciferase activity in HEK293T cells co-transfected with RIG-I, MAVS, TBK1, IKKE, or IRF3(5D) and Siglec1 as indicated. (B) Immunoblot analysis of the indicated molecules in lysates of macrophages transfected with Siglec1 siRNA\#1 and infected with VSV (MOI = 10). (C) Immunoblot and RT-PCR analyses were performed to respectively measure TBK1 protein and mRNA levels in macrophages transfected with Siglec1 siRNA and stimulated with poly(l:C) transfection at the indicated time. Actin serves as a loading control. (D) Immunoblot and RT-PCR analyses were performed to respectively measure TBK1 protein and mRNA levels in RAW264.7 cells transfected with increasing doses of Siglec1 plasmid. Data are shown as mean \pm SD or representative photographs. ${ }^{* *} P<0.01$; NS, not significant. See also Supplementary information, Figure S3.

with DAP12 and FcR $\gamma$, but not DAP10 (Figure 4D). We also found that DAP12 associated with Siglec1 and SHP2, and SHP2 associated with Siglec1 and DAP12, suggesting that Siglec1, DAP12, and SHP2 form a complex (Figure 4E). Furthermore, SHP1 did not exist in the complex (Figure 4E). Interestingly, overexpression of DAP12 was found to promote the binding between Siglec1 and SHP2 (Figure 4E). Collectively, these data suggest that upon VSV infection, Siglec1 may associate with DAP12 and SHP2, thus leading to suppression of type I IFN production.

SHP2 recruits TRIM27 to suppress VSV-triggered type I IFN production

Among Siglec1-associated proteins detected by MS, we focused on an E3 ligase TRIM27 to examine its potential role in regulating TBK1 turnover (Supplementary information, Figure S4). We did not find any direct interaction between Siglec1 and TRIM27 in HEK293T cells overexpressing these two proteins (data not shown), but IP and GST pull-down experiments showed that SHP2 directly interacted with TRIM27 (Figure 5A and Supplementary information, Figure S6A). Furthermore, enhanced interaction among endogenous SHP2, TRIM27, and DAP12 was observed upon VSV infection (Figure $5 B)$. Also, co-IP experiments revealed that SHP2 associated with TRIM27 and DAP12, and DAP12 associated with TRIM27 and SHP2 in HEK293T cells co-expressing various tagged plasmids (Figure 5C and Supplementary information, Figure S5A). These results suggest that 
A
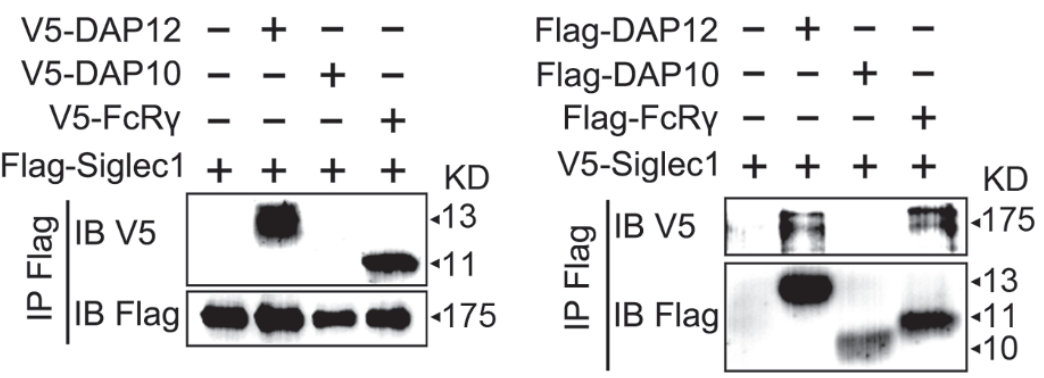

B

(h) $\frac{\mathrm{VSV}}{0 \quad 2 \quad 4} \operatorname{lgg} \frac{\mathrm{HSV}}{0 \quad 4}$

$$
\text { C }
$$

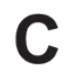

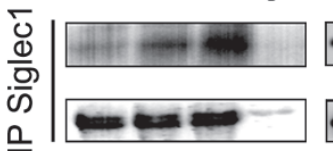
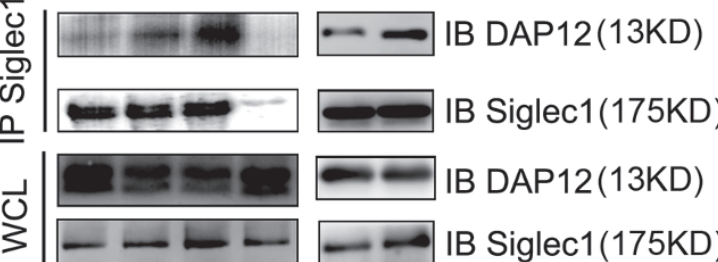

IB DAP12(13KD)

IB Siglec1(175KD)

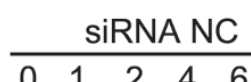

\section{siSiglec1}

\begin{tabular}{lllllllllllllll}
\cline { 3 - 5 } Time(h) & 0 & 1 & 2 & 4 & 6 & 8 & 0 & 1 & 2 & 4 & 6 & 8 & $\mathrm{KD}$
\end{tabular}

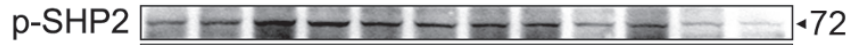

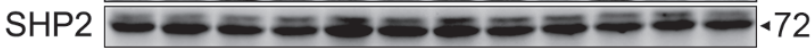

p-Syk - - - - - $-\longrightarrow-\cdots-17$

Syk - - - -

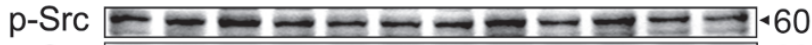

Src $-2-2-160$

p-SHP1 - - - - - - - - - - - -

SHP1 - 68

$\beta$-Actin WDat.43

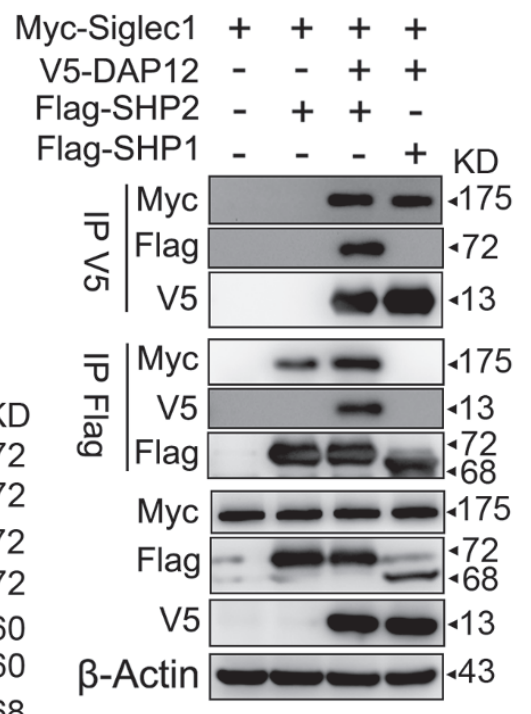

D

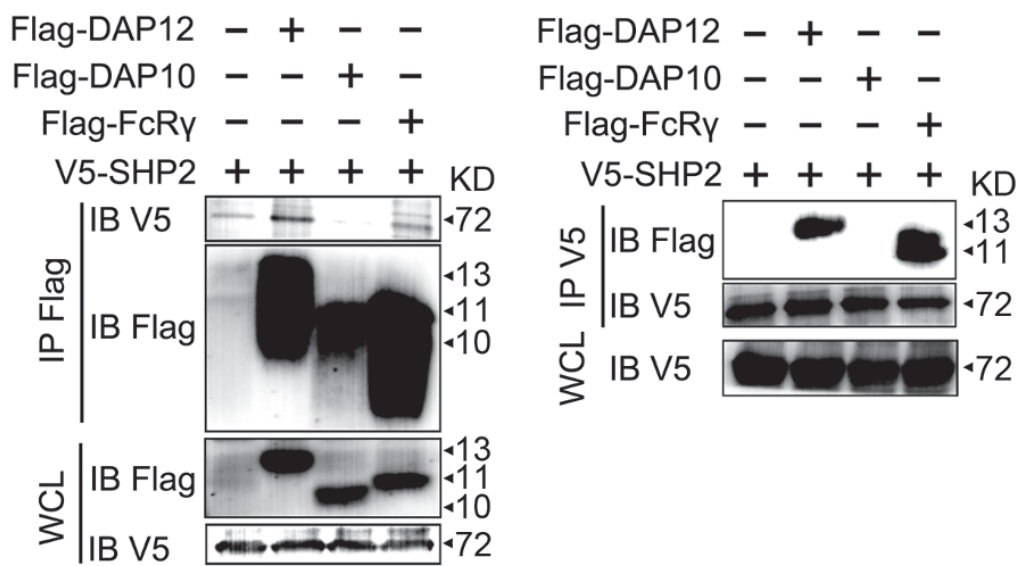

Figure 4 Siglec1 associates with DAP12 and SHP2 in VSV-infected macrophages. (A) IP and immunoblot analyses of the indicated proteins in HEK293T cells co-transfected with Siglec1 and DAP12, DAP10, or FcR $\gamma$ as indicated. (B) IP and immunoblot analyses of the endogenous DAP12-Siglec1 interaction in macrophages infected with VSV or HSV. IgG served as an IP control. (C) Immunoblot analysis of the indicated molecules in macrophages transfected with NC or Siglec1 siRNA and infected with VSV $(\mathrm{MOI}=10)$. (D) IP and immunoblot analyses of the indicated proteins in HEK293T cells co-transfected with SHP2 and DAP12, DAP10, or FcR $\gamma$ as indicated. (E) IP and immunoblot analyses of the indicated proteins in HEK293T cells co-transfected with Siglec1 and DAP12, SHP2, or SHP1 as indicated. Data are shown as representative photographs. See also Supplementary information, Figure S4. 
DAP12, SHP2, and TRIM27 may form a complex and play important roles in Siglec1-mediated signaling pathway.

We further investigated whether TRIM27 plays a role in Siglec1-mediated regulation of type I IFN production. First, we found that TRIM27 knockdown significantly inhibited VSV replication and increased virus-triggered type I IFN production (Figure 5D and 5E). We established TRIM27-overexpressing RAW264.7 macrophages (Figure 5F), and found that type I IFN production induced by VSV or HSV infection was inhibited and VSV replication was enhanced in these cells (Figure 5G), further confirming that TRIM27 could inhibit type I IFN production. Moreover, TRIM27 knockdown enhanced IRF3 and TBK1 phosphorylation, while phosphorylation of ERK, p38, JNK, and p65 was less influenced (Figure $5 \mathrm{H})$. We also found that overexpression of Siglec 1 or TRIM27 reduced TBK1-induced IFN- $\beta$ luciferase activation, and overexpression of both further inhibited IFN- $\beta$ reporter activation. However, overexpression of both Siglec1 and TRIM27 failed to reduce the constitutively active IRF3(5D)-induced IFN- $\beta$ luciferase activation (Figure 5I), suggesting that Siglec1 and TRIM27 have cumulative effects on downregulating type I IFN production, which is dependent on TBK1.

To further investigate the role of SHP2 in Siglecl-mediated signaling pathway, we used SHP2-deficient macrophages and found that Siglec1 knockdown failed to upregulate VSV infection-induced IFN- $\beta$ production in SHP2-deficient macrophages (Figure 5J). Although TRIM27 knockdown still facilitated VSV-induced IFN- $\beta$ production in SHP2-deficient macrophages, such facilitating effect was smaller as compared with that in WT macrophages (Supplementary information, Figure $\mathrm{S} 5 \mathrm{C})$. These results suggest that SHP2 is required for the inhibitory effects of Siglec 1 and TRIM 27 on IFN- $\beta$ production, and SHP2 may function upstream of TRIM27 and downstream of Siglec1. We next examined whether SHP2 phosphorylation is required for Siglecl-mediated signaling. Intriguingly, WT SHP2, catalytically inactive SHP2 (SHP2CS), and SHP2 phosphorylation-defective mutant (SHP2 Y542A/Y580A) are all capable of decreasing VSV-triggered IFN- $\beta$ production in SHP2-deficient cells (Supplementary information, Figure S5B). We also found that SHP2CS was still able to associate with TRIM27 (Supplementary information, Figure S5D). These results indicate that SHP2 negatively regulates type I IFN production in a phosphatase activity- and phosphorylation-independent manner. Furthermore, Siglec1 failed to inhibit IFN- $\beta$ production in macrophages with TRIM27 knockdown (Figure 5K), but could still do so in cells with knockdown of other reported TBK1 E3 ligase DTX4 [9] or TRIP [20] (Supplementary informa- tion, Figure S5E), suggesting that TRIM27 plays a key role in Siglec1-mediated inhibition of type I IFN production. Together, these results indicate that upon VSV infection, Siglec1 activates the scaffolding function of SHP2, and then SHP2 recruits TRIM27 to work jointly to suppress type I IFN production.

\section{TRIM27 inhibits VSV infection-induced type I IFN pro-} duction by promoting TBK1 degradation

We previously reported that SHP2 can interact with TBK1 and inhibit TBK1-mediated IFN production, but the mechanism is unknown [25]. We then investigated whether SHP2 recruits TRIM27 to TBK1 for inhibiting VSV-triggered type I IFN production. The direct association between TRIM27 and TBK1 was detected (Figure 6A and Supplementary information, Figure S6A) and the co-localization between TRIM27 and TBK1 upon VSV infection was observed using confocal microscopy (Supplementary information, Figure S6B). Overexpression of TRIM27 decreased TBK1 protein level, while knockdown of TRIM27 increased TBK1 protein level (Figure 6B and Supplementary information, Figure S6C). We also found that TBK1 mRNA level did not change upon overexpression of TRIM27 (Figure 6C), thus excluding the possibility that the decrease of TBK1 protein level was caused by lower expression of the TBK1 gene. These data suggest that TRIM27 may function primarily as an E3 ubiquitin ligase to degrade TBK1. The proteasome inhibitor MG-132 blocked TRIM27-induced decrease of TBK1 protein level (Figure 6D), indicating that TRIM27 decreases TBK1 expression by inducing TBK1 degradation via the proteasome pathway. In characterization of TRIM27-mediated ubiquitination of TBK1, we found that TRIM27 induced an increase in K48-linked ubiquitination of TBK1, whereas K63-linked ubiquitination remained unchanged (Figure 6E), suggesting that TRIM27 induces K48-linked polyubiquitination of TBK1 to facilitate its degradation. It was reported that phosphorylation of TBK1 at serine 172 was critical for NLRP4-mediated ubiquitination of TBK1 [9]. We found that TRIM27 was still able to bind and induce K48-linked ubiquitination of the TBK1 S172A mutant (Supplementary information, Figure S6D), indicating that TRIM27-induced TBK1 degradation may be independent of TBK1 Ser172 phosphorylation. Together, these results suggest that TRIM27 functions as an E3 ubiquitin ligase of TBK1 and promotes its degradation.

In order to characterize the TRIM27-TBK1 interaction in more detail, we constructed plasmids expressing various truncated fragments of TRIM27 and TBK1. Binding between TRIM27 and TBK1 was mediated by the interaction between their respective coiled-coil domains, and 
A

$$
\begin{aligned}
& \text { V5-SHP2 }++ \\
& \text { Flag-TRIM27 - + }
\end{aligned}
$$

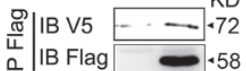

$$
\begin{aligned}
& \text { 仓IB V5 } \\
& \text { Flag-TRIM27 }++ \\
& \text { V5-SHP2 - + } \\
& \text { ๖|IB Flag } \longrightarrow .58 \\
& \text { a IB V5 }
\end{aligned}
$$

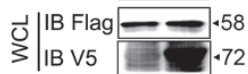

B

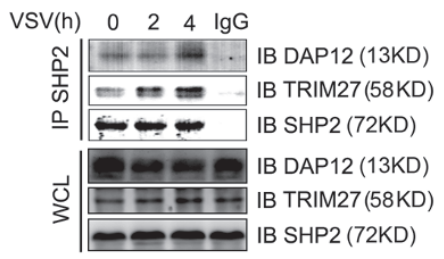

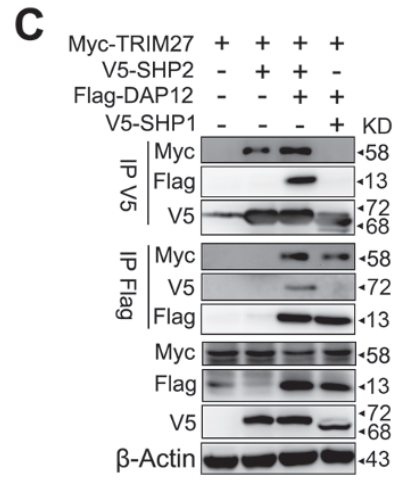

D

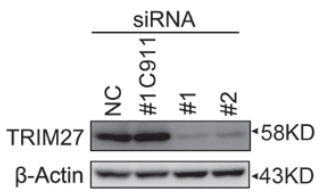

F

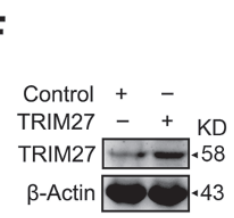

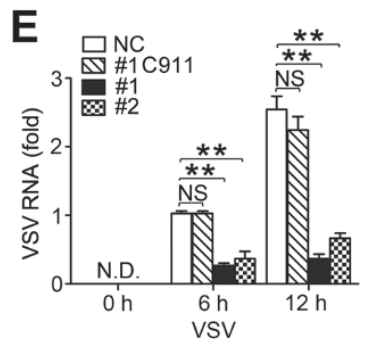
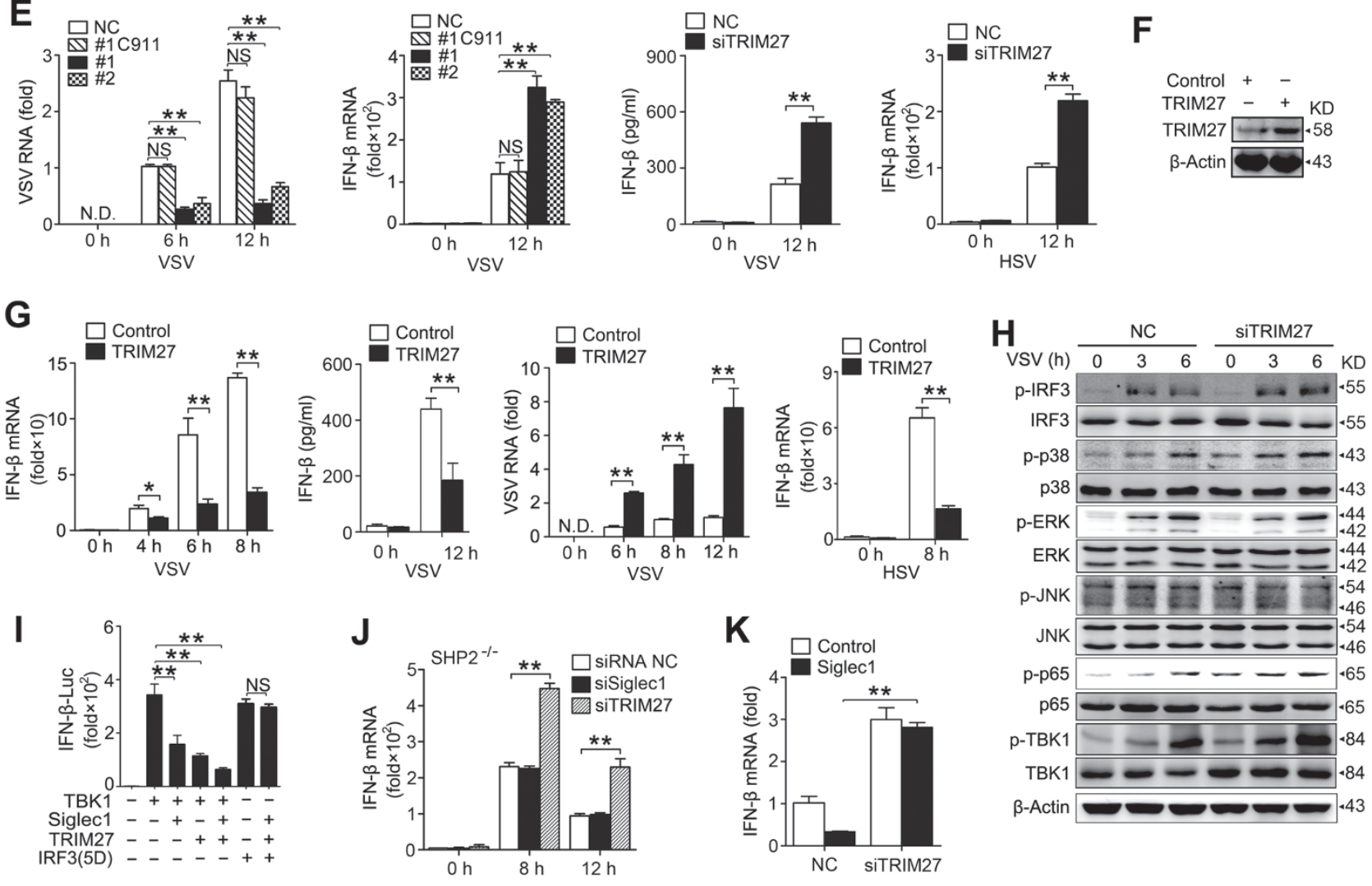

Figure 5 Recruitment of TRIM27 by SHP2 inhibits VSV-triggered type I IFN production in macrophages. (A) IP and immunoblot analyses of the indicated proteins in HEK293T cells co-transfected with TRIM27 and SHP2 as indicated. (B) Immunoblot analysis of DAP12 and TRIM27 in SHP2-immunoprecipitated products from lysates of VSV-infected $(\mathrm{MOI}=10)$ macrophages. (C) IP and immunoblot analyses of the indicated proteins in HEK293T cells co-transfected with TRIM27 and DAP12, SHP2, or SHP1 as indicated. (D) Immunoblot analysis of TRIM27 in macrophages transfected with TRIM27 siRNA as indicated for $48 \mathrm{~h}$. (E) Macrophages were transfected as in $\mathbf{D}$ and then infected with VSV $(\mathrm{MOI}=10)$ or $\mathrm{HSV}(\mathrm{MOI}=10)$ for $12 \mathrm{~h}$. Q-PCR analysis was performed to evaluate IFN- $\beta$ and VSV RNA levels in cells, and ELISA was performed to evaluate IFN- $\beta$ protein levels in the supernatants. (F) Immunoblot analysis of TRIM27 in RAW264.7 cell clones stably overexpressing TRIM27. (G) RAW264.7 cell clones stably overexpressing TRIM27 were infected with VSV $(\mathrm{MOI}=10)$ or $\mathrm{HSV}(\mathrm{MOI}=10)$ for the indicated time. Q-PCR analysis of intracellular IFN- $\beta$ mRNA and VSV RNA levels and ELISA of IFN- $\beta$ in the supernatants were then performed. $(\mathrm{H})$ Immunoblot analysis of the indicated molecules in lysates of macrophages transfected with TRIM27 siRNA\#1 and infected with VSV (MOI = 10). (I) IFN- $\beta$ luciferase activity in HEK293T cells transfected with TBK1 or IRF3(5D), and Siglec1 or TRIM27 as indicated. (J) Q-PCR analysis of IFN- $\beta$ mRNA levels in SHP2-deficient macrophages transfected with Siglec1 or TRIM27 siRNA and infected with VSV. (K) Q-PCR analysis of IFN- $\beta$ mRNA levels in Siglec1 stably overexpressing-RAW264.7 cell clones that were transfected with TRIM27 siRNA and infected with VSV for $8 \mathrm{~h}$. Data are shown as mean \pm SD or representative photographs. ${ }^{*} P<0.05$; ${ }^{* *} P<0.01$; NS, not significant. See also Supplementary information, Figure S5. 

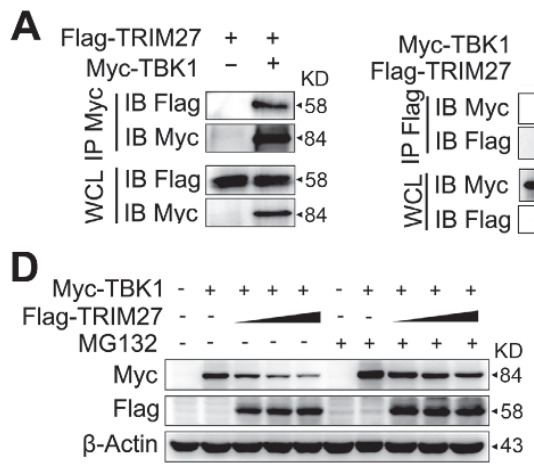

E

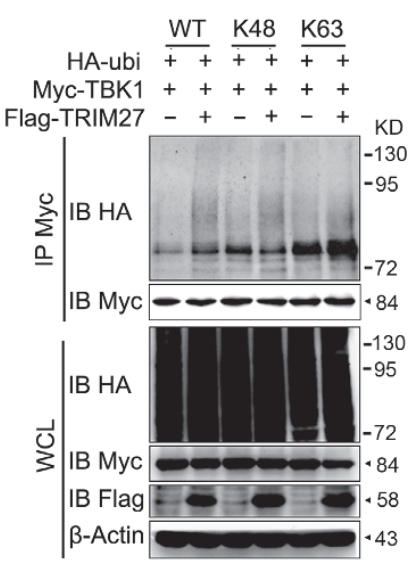

B

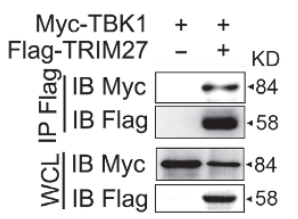

$\mathbf{F}$

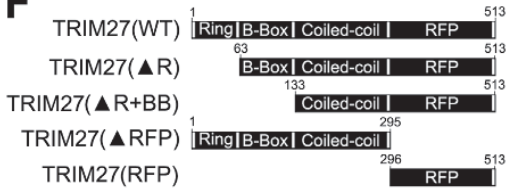

TRIM27(RFP)

Flag-TRIM27(WT) - + - - - -

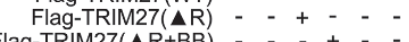

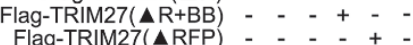
Flag-TRIM27(RFP) -----+ Myc-TBK $1++++++$ KD
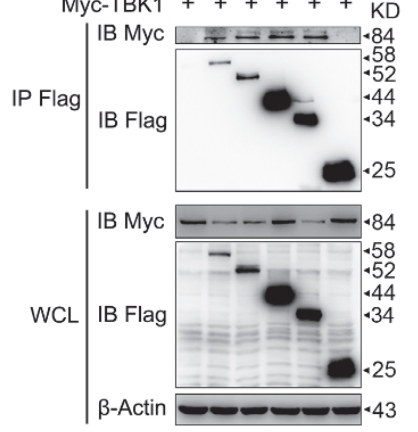

C

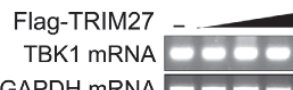

GAPDH mRNA $-\infty$

G

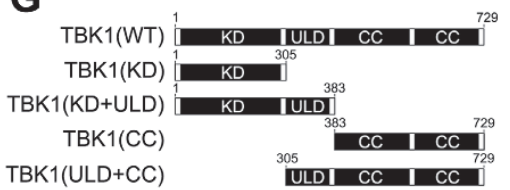

Myc-TBK1 $(\mathrm{WT})+\ldots$

Myc-TBK1(KD) - + - - -

Myc-TBK1 (KD+ULD) - - + - -

Myc-TBK1(CC) - - - + -

Myc-TBK1(ULD+CC) - - - - +

Flag-TRIM27 +++++ KD
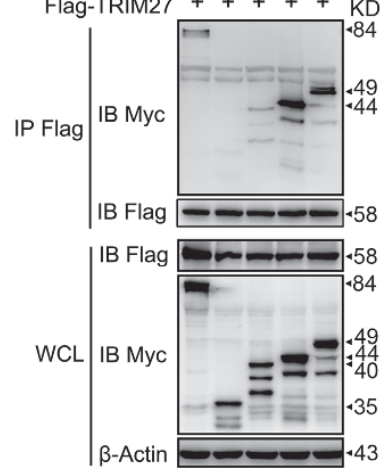

Figure 6 TRIM27-mediated K48-linked ubiquitination and degradation of TBK1. (A) IP and immunoblot analyses of the indicated proteins in HEK293T cells co-transfected with TRIM27 and TBK1 as indicated. (B) Immunoblot analysis of TBK1 in RAW264.7 cells transfected with increasing doses of TRIM27 plasmids. (C) RT-PCR analysis of TBK1 mRNA levels in RAW264.7 cells transfected with increasing doses of TRIM27 plasmids. GAPDH mRNA serves as a loading control. (D) Immunoblot analysis of extracts from HEK293T cells transfected with TBK1 and TRIM27 plasmids and treated with MG132 or dimethyl sulfoxide (DMSO). (E) IP and immunoblot analyses of extracts from HEK293T cells transfected with TBK1 with or without TRIM27 as well as WT, K48-linked, or K63-linked ubiquitin as indicated. (F) IP and immunoblot analyses of the indicated proteins in HEK293T cells transfected with various TRIM27 truncated fragments along with TBK1. $\mathbf{R}$, lacking RING domain; $\Delta \mathrm{R}+\mathrm{BB}$, lacking RING and B-Box domains; $\Delta$ RFP, lacking PRY-SPRY domain; RFP, PRY-SPRY domain. Numbers above the domain names indicate amino acid positions. (G) IP and immunoblot analyses of the indicated proteins in HEK293T cells transfected with various TBK1 truncated fragments and TRIM27 as indicated. KD, kinase domain; ULD, ubiquitin-like domain; CC, coiled-coil domain. Data are shown as representative photographs. See also Supplementary information, Figure S6.

the B-Box domain of TRIM27-mediated TBK1 degradation (Figure 6F and 6G). We also found that TRIM27 mutants lacking the B-Box domain were no longer able to inhibit TBK1-induced IFN- $\beta$ luciferase activation, while TRIM27 fragments containing the B-Box domain retained this function (Supplementary information, Figure S6E). Therefore, the coiled-coil and B-Box domains of TRIM27 are responsible for TBK1 interaction and degradation, respectively.

\section{TRIM27 induces K48-linked ubiquitination of TBK1 at} Lys 251 and Lys 372

We further attempted to identify the specific sites of TRIM27-mediated ubiquitination in TBK1. Among all
TBK1 truncations examined, the fragment containing only the coiled-coil domain did not show enhanced K48linked ubiquitination and degradation upon TRIM27 overexpression (Figure 7A and 7B), suggesting that the kinase domain and the ubiquitin-like domain of TBK1 may contain sites for TRIM27-mediated ubiquitination. Using computer-assisted algorithms [26], we identified five potential ubiquitination sites (Lys82, Lys241, Lys251, Lys341, and Lys372) in these two domains, and substituted them with arginine to create five single-site mutants and a double-site mutant K251/372R. We found that the K251/372R double mutation almost completely blocked TRIM27-induced degradation and K48-linked ubiquitination of TBK1 (Figure 7C and 7D), suggesting 
that K251 and K372 are required for TRIM27-mediated TBK1 ubiquitination. Furthermore, the inhibitory effect of TRIM27 on TBK1-induced IFN- $\beta$ luciferase activation was reduced by either K251R or K372R single mutation of TBK1, and was completely abolished by the K251/372R double mutation (Figure 7E). Siglec1 was unable to inhibit IFN- $\beta$ luciferase activation induced by the K251/372R mutant of TBK1 (Figure 7F). In addition, promotion of VSV replication induced by overexpression of Siglec1 or TRIM27 was abolished by transfection of the K251/372R TBK1 mutant (Figure 7G). However, the K251/372R mutant can still bind to TRIM27 (Supplementary information, Figure S7). Taken together, these results suggest that Lys 251 and Lys372 of TBK1 are essential for Siglec1- and TRIM27-mediated K48-linked ubiquitination and degradation of TBK1. Hence, our results reveal a negative feedback pathway regulating type I IFN production, in which IFN-induced upregulation of Siglec1 in turn activates TRIM27-mediated TBK1 degradation (Figure 7H and 7I).

\section{Discussion}

Although an effective host defense against viral infection requires the induction of appropriate innate immune responses, excessive activation of antiviral innate immune responses may cause the development of acute or chronic inflammatory disorders. Therefore, immune cells need to employ various negative regulatory mechanisms to avoid uncontrolled immune responses when encountering viral invasion. Here we discovered that inducible upregulation of the Siglec1 pathway and increase of TBK1 degradation may represent a new feedback regulatory mechanism for the host antiviral immune response.

Siglec1 is expressed in many types of immune cells with a preferential expression in macrophages [27]. In contrast, IFNAR is extensively expressed in many tissues and cell types, and highly expressed in immune cells. As innate immune cells, especially macrophages and DCs, are important IFN-producing cells, the negative regulation of IFN production in these cells is important for the prevention of excessive IFN production-mediated immune disorders. We now demonstrate that type I IFN can induce Siglec1 expression in macrophages and DCs, suggesting that the negative regulatory loop of type I IFN production mediated by Siglec1 exists in uninfected cells and can be induced upon viral infection in innate immune cells. Hence, the activated Siglec1 pathway by type I IFN may represent a new mechanism to prevent excessive type I IFN production.

Excessive IFN production has been suggested to be involved in the pathogenesis of a set of autoimmune diseases, especially the systemic lupus erythematosus [28]. In these diseases, negative regulators or regulatory pathways of IFN production are often deregulated or inactivated. Here we show that Siglec1 expression can be significantly induced by type I IFN, and the induced Siglec1 in turn suppresses IFN production. Siglec1-mediated negative feedback loop controlling IFN production may be important for the prevention of IFN-mediated autoimmune diseases. It would be interesting to further investigate whether the Siglec1 pathway is deregulated in autoimmune diseases. Host negative regulation of type I IFN production may also be exploited by the virus to subvert the immune responses, thus helping virus to promote its replication and immune evasion in host cells. Siglecs are inhibitory receptors defined by their ability to recognize sialic-acid-containing structures. Several types of pathogens can exploit sialic acids, which are either synthesized by themselves or acquired from the host, to activate inhibitory Siglecs on innate immune cells, thus attenuating host protective responses and achieving immune evasion [11]. Membrane Siglec1 has been reported to be utilized by HIV-1 to infect macrophages [29]. Together with our data that Siglec1 expression was upregulated upon viral infection, we thus speculate that Siglec1 upregulation may be an important and common strategy utilized by different families of viruses to evade host immune responses. Therefore, Siglec1 may bear considerable potential to serve as a new target for the development of therapies against viral infection and infection-related diseases.

Siglec1 is expressed in numerous macrophage populations and contains an extended extracellular region consisting of 17 Ig-like domains. It is believed to primarily function in mediating macrophage-pathogen adhesion and phagocytosis [30] as well as in promoting antigen presentation [31]. Siglec1 does not contain any known cytosolic motifs to mediate inhibitory signaling itself, and the interaction between Siglec1 and intracellular regulators is poorly understood. Here we found that Siglec1 associates with DAP12 to activate the scaffolding function of SHP2, which then recruits TRIM27 to degrade TBK1. We have previously reported that Siglec-G recruits the E3 ligase c-Cbl to degrade RIG-I via K48linked ubiquitination [10]. Therefore, both Siglec1 and Siglec-G pathways can be utilized by viruses to evade host immune responses and act in parallel by targeting different key signaling molecules of innate immune responses. Our findings have provided new insight into mechanisms by which membrane-bound Siglec family members regulate intracellular signaling pathways. However, the interaction mechanism between Siglec1 and DAP12 still needs to be investigated in the future. 


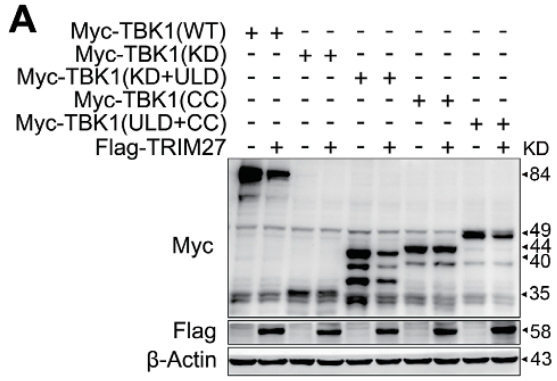

B

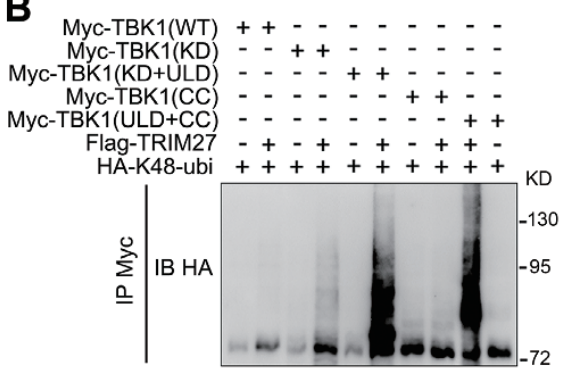

$\mathbf{E}$
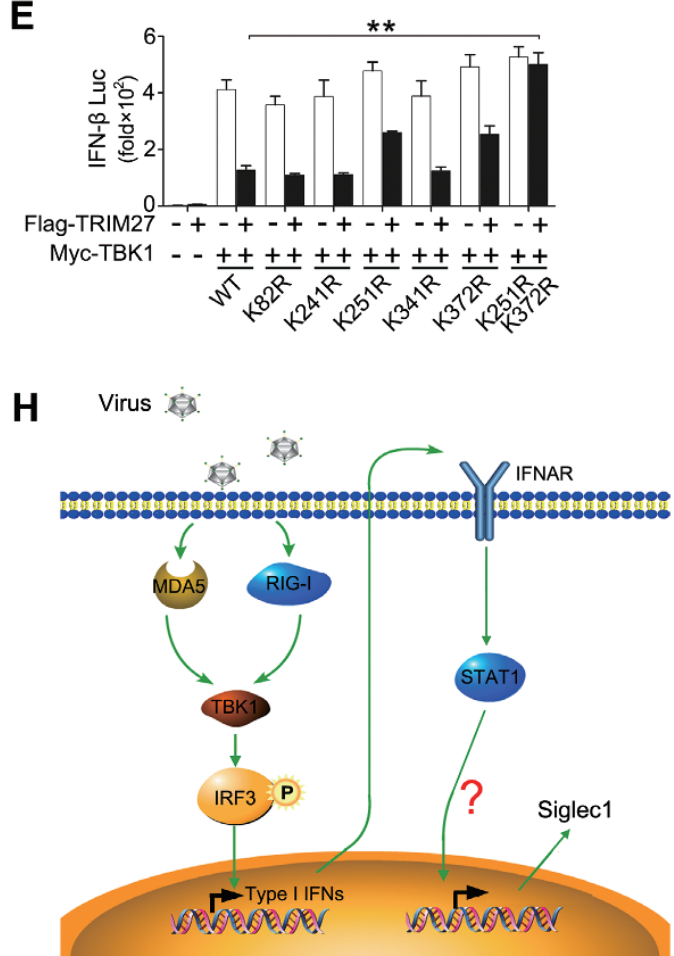

C
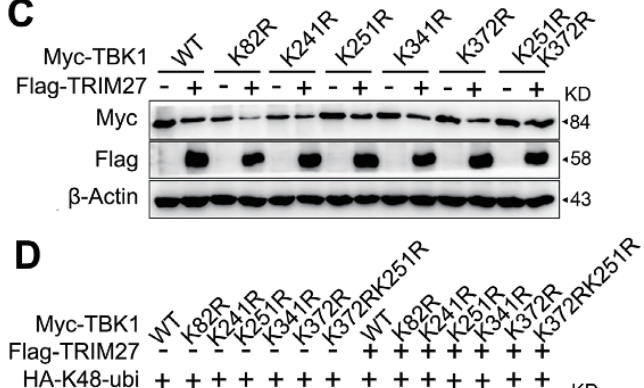

HA-K48-ubi $t+++++++++++++{ }_{\mathrm{KD}}$

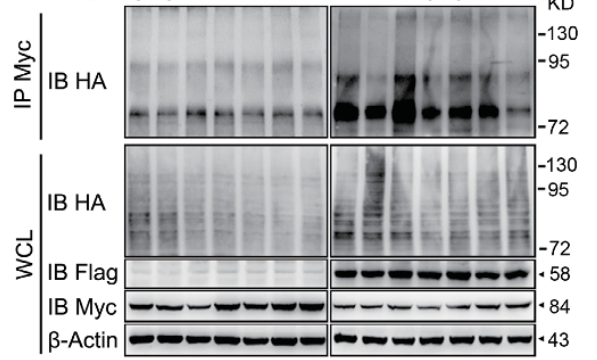

$\mathbf{F}$
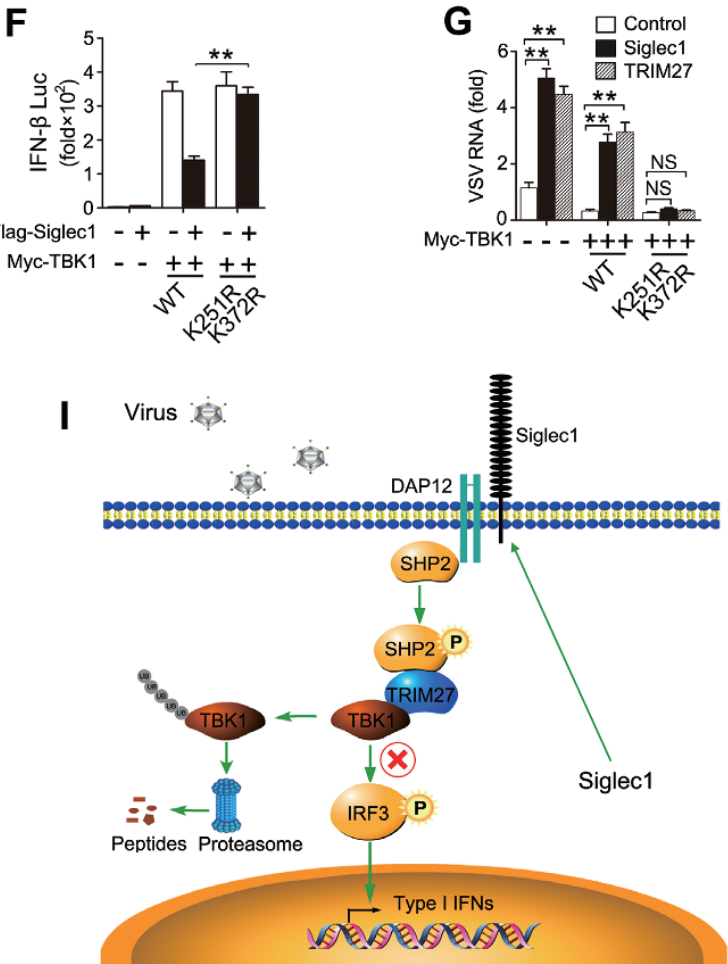

Figure 7 Lys251 and Lys372 of TBK1 are critical sites for TRIM27-mediated degradation of TBK1. (A, B) IP and immunoblot analyses of the indicated proteins in HEK293T cells co-transfected with various TBK1 truncated fragments (KD, 35 KD; KD+ULD, 40 KD; CC, 44 KD; ULD+CC, 49 KD), TRIM27, and K48-linked ubiquitin as indicated. (C, D) IP and immunoblot analyses of the indicated proteins in HEK293T cells transfected with TRIM27 and K48-linked ubiquitin together with various TBK1 mutants as indicated. (E) IFN- $\beta$ luciferase activity in HEK293T cells transfected with various TBK1 mutants and TRIM27. (F) IFN- $\beta$ luciferase activity in HEK293T cells transfected with WT or mutant TBK1 and Siglec1 as indicated. (G) Q-PCR analysis of VSV RNA levels in RAW264.7 cells that were transfected with WT or mutant TBK1 and Siglec1 or TRIM27 and then infected with VSV $(\mathrm{MOI}=10)$ for $12 \mathrm{~h}$. (H, I) Working model for the mechanism by which viral infection-induced upregulation of Siglec1 negatively regulates type I IFN production in a feedback manner via recruiting TRIM27 to degrade TBK1 and impair IRF3 signaling in the initiation of type I IFN production. This proposed negative feedback pathway is independent of the phosphorylation and the phosphatase activity of SHP2. Data are shown as mean \pm SD or representative photographs. ${ }^{* *} P<0.01$. See also Supplementary information, Figure S7. 
Ubiquitination is one of the most versatile PTMs and is important for antiviral responses. Many members of the TRIM family can induce K63- or K48-linked ubiquitination [32]. For example, TRIM25 and TRIM56 can induce RIG-I and STING ubiquitination, respectively, by K63-mediated linkage, which are essential for type I IFN production $[33,34]$. In contrast, TRIM21 negatively regulates type I IFN production mainly through promoting K48-linked ubiquitination and degradation of DDX41 [35]. Recently, TRIM27 was described to act as an E3 ligase that negatively regulates NOD2 by mediating its ubiquitination and proteasomal degradation [36]. Here we show that TRIM27 is a key E3 ligase that mediates K48-linked ubiquitination at Lys251 and Lys372 of TBK1 and promotes TBK1 proteasomal degradation, thus functioning as a negative regulator of type I IFN production. It was reported that Lys620 is the site for TBK1 ubiquitination by DTX4, suggesting that different E3 ligases could target TBK1 for degradation during viral infection. More importantly, knockdown of TRIM27 blocked Siglec1-mediated inhibition of type I IFN production, but knockdown of other reported TBK1 E3 ligases, DTX4 or TRIP, in macrophages failed to do so. Furthermore, DTX4 and TRIP were not detected by MS as Siglec1-associated proteins (data not shown). These data suggest that TRIM27 serves as a specific E3 ligase for TBK1 in the Siglec1-mediated signaling pathway during viral infection. The RING domain of TRIM family members was reported to mediate their E3 ubiquitin ligase activity; however, we found that TRIM27-mediated TBK1 degradation is independent of its RING domain and is instead dependent on its B-Box domain. Why only the B-Box domain of TRIM27 is involved in TBK1 ubiquitination still needs to be investigated in the future.

SHP-2 is an evolutionarily conserved protein tyrosine phosphatase, containing two $\mathrm{SH} 2$ domains at the $\mathrm{N}$ terminus, a central catalytic domain, and a C-terminal tail. SHP-2 functions as a regulator in various signaling pathways [37]. Here we show that SHP2 negatively regulates type I IFN production in a phosphatase activityand phosphorylation-independent manner. We also found that SHP2 is required for TRIM27-mediated negative regulation of IFN production. Hence, we propose that SHP2 may function as an adaptor between DAP12 and TRIM27. However, the detailed mechanisms of SHP2 activation and its role in TRIM27 activation still need further investigation.

On the basis of our findings, we propose the following working model to explain how inducible upregulation of Siglec1 inhibits type I IFN production and suppresses the antiviral innate immune response (Figure $7 \mathrm{H}$ and $7 \mathrm{I}$ ). Viral infection significantly upregulates Siglec1 expression in macrophages through the IFN/JAK/STAT1 signaling pathway. Siglec1 in turn inhibits type I IFN production through targeting TBK1. Mechanistically, Siglec1 associates with DAP12 and activates the scaffolding function of SHP2; SHP2 subsequently recruits TRIM27, which then binds TBK1 through an interaction between their coiled-coil domains. TRIM27 then functions as an E3 ligase to induce K48-linked ubiquitination at both Lys251 and Lys372 residues of TBK1, consequently leading to proteasomal degradation of TBK1, suppression of IRF3 phosphorylation, and inhibition of type I IFN production.

\section{Materials and Methods}

\section{Mice}

C57BL/6 mice (6-8 weeks) were obtained from the Joint Ventures Sipper BK Experimental Animal Company (Shanghai, China). IFNAR-deficient mice were obtained from Jackson Laboratories (Bar Harbor, ME, USA). SHP2-deficient mice were a kind gift from Dr Gensheng Feng (University of California San Diego, San Diego, CA, USA). Mice were housed and bred in specific pathogen-free conditions. All animal experiments were performed in accordance with the National Institute of Health Guide for the Care and Use of Laboratory Animals, with the approval of the Scientific Investigation Board of Second Military Medical University (Shanghai, China).

\section{Cell culture and transfection}

HEK293T and RAW264.7 cell lines were obtained from American Type Culture Collection (ATCC, Manassas, VA, USA) and cultured as described previously [38]. Cells $\left(1 \times 10^{6}\right)$ were seeded into 6-well plates and incubated overnight. Jet-PRIME transfection reagents (Polyplus-transfection, Illkirch, France) were used for co-transfection of plasmids and siRNAs according to the manufacturer's instructions. Thioglycollate-elicited mouse peritoneal macrophages were seeded $\left(2 \times 10^{5}\right.$ cells in $\left.0.5 \mathrm{ml}\right)$ into 24 -well plates and incubated overnight. Cells were then transfected with siRNAs using INTERFERin (Polyplus-transfection) according to the manufacturer's instructions. To establish stably transfected RAW264.7 cells, G418 was added ( $1000 \mu \mathrm{g} / \mathrm{ml}) 48 \mathrm{~h}$ after transfection and maintained at $800 \mu \mathrm{g} / \mathrm{ml}$ for 3 weeks for positive selection. For stably transfected cells, Siglec1 and TRIM27 expression was confirmed by western blot. Stably transfected RAW264.7 cells were subsequently cultured in a complete medium containing $500 \mu \mathrm{g} / \mathrm{ml}$ G418.

\section{Reagents and pathogens}

The STAT1 inhibitor (Fludara, $100 \mu \mathrm{g} / \mathrm{ml}$ ), GSK inhibitor (SB216763, $10 \mu \mathrm{M})$, JNK inhibitor (SP1006, $30 \mu \mathrm{M}), \mathrm{NF}-\kappa \mathrm{B}$ inhibitor (PDTC, $200 \mu \mathrm{M})$, and MG132 $(5 \mu \mathrm{M})$ were purchased from Calbiochem (San Diego, CA, USA). Poly(I:C) (P1530) and glutathione (G4251) were purchased from Sigma-Aldrich (St. Louis, MO, USA). rIFN- $\alpha 1 b$ was obtained from Sanyuan Company (Shanghai, China). Antibodies against $\beta$-actin (sc-8432) and DAP12 (sc-20783), and horseradish peroxidase (HRP)-coupled secondary antibodies (sc-2749) were from Santa Cruz Biotechnology (Santa Cruz, CA, USA). Antibody against Siglec1 (MAB5610) 
was from R\&D Systems (Minneapolis, MN, USA). Antibodies against STAT1 (9172), p-IRF3 (4947), IRF3 (4302), p-JNK (9251), JNK (9252), p-p65 (3033), p65 (8242), p-p38 (9215), p38 (9212), p-Src (2113), Src (2123), p-SHP2 (3751), SHP2 (3752), p-Syk (14140), Syk (13198), p-SHP1(8849), SHP1(3759), p-TBK1 (5483), TBK1 (3013), and Myc-tag (2278) were from Cell Signaling Technology (Danvers, MA, USA). Antibodies against HA (ab9110) and V5 (ab9116) were from Abcam (Cambridge, UK). Antibodies against TRIM27 (AV34701) and the Flag-tags (F7425) as well as the agarose used for IP were from Sigma-Aldrich. HRP-conjugated secondary antibody (TrueBlot; 18-8816) was from eBioscience (San Diego, CA, USA). Alexa Fluor 488 (A-21202) and Alexa Fluor 546 (A-11071) were from Life technologies. VSV (Indiana Strain) was propagated and amplified by infecting a monolayer of Vero cells. The supernatant was harvested $24 \mathrm{~h}$ later and clarified by centrifugation. Viral titers were determined by evaluating TCID $_{50}$ levels in Vero cells. Sendai virus and HSV-1 virus (Kos strain) were kindly provided by Dr Qihan Li (Chinese Academy of Medical Sciences).

\section{RNA interference}

Thioglycollate-elicited mouse peritoneal macrophages or RAW264.7 cells were transfected with siRNA (final concentration: $20 \mathrm{nM}$ ) using the INTERFERin reagent (Polyplus-transfection). All siRNAs were obtained from GenePharma (Supplementary information, Table S1).

\section{Real-time quantitative PCR}

Total RNA was extracted with Trizol reagent (Invitrogen) and reversed-transcribed using the Reverse Transcription System from Toyobo (Osaka, Japan). The reverse transcription products from different samples were amplified by real-time PCR and analyzed as described previously [39, 40]. The primer sequences for Q-PCR analysis are listed in Supplementary information, Table S2.

\section{Molecular cloning of related genes}

Related genes were obtained from mouse macrophage or HEK293T cDNA by RT-PCR and subsequently subcloned into pGL3-enhancer, pcDNA-Flag, pcDNA-Myc, or pcDNA-V5 vectors. The deletion domains of mouse Flag-TRIM27 and MycTBK1 were generated by PCR. Corresponding primers used are listed in Supplementary information, Table S3. Phosphorylation and ubiquitination site point mutations in TBK1 were generated by PCR-based amplification. Each construct was confirmed by sequencing. The corresponding primers used in this study are listed in Supplementary information, Table S4.

Nanospray liquid chromatography-tandem mass spectrometry Macrophages were infected with VSV for $6 \mathrm{~h}$ or uninfected (0 h), and then lysed for immunoprecipitation with Siglec1 antibody. After silver staining, different bands with intensive signal in $6 \mathrm{~h}$ compared with $0 \mathrm{~h}$ and IgG control were cut and digested, followed by analysis by reverse-phase nanospray liquid chromatography-tandem mass spectrometry. The spectra from tandem mass spectrometry were automatically used for searching against the nonredundant International Protein Index mouse protein database (version 3.72) with the Bioworks browser (rev.3.1).

\section{Confocal microscopy}

HEK293T cells plated on glass coverslips in six-well plates were co-transfected with plasmids and infected with VSV. Cells were then labeled with tag antibody and viewed using a Leica TCS SP2 confocal laser microscope.

\section{GST pull-down assays}

The cDNAs encoding TRIM27 were cloned into pGEX-4T-1 vector (GE Healthcare), and cDNAs encoding SHP2, TBK1, or SHP1 were cloned into pET-22b(+) vector (Novagen). The expression and purification of GST or $6 \times$ His fusion proteins and the GST pull-down assays were performed as previously described [25].

\section{Luciferase reporter assays}

HEK293T cells $\left(1 \times 10^{4}\right)$ were plated in 96-well plates and transfected with a mixture of the indicated luciferase reporter plasmid and the pRL-TK-Renilla luciferase plasmid together with various amounts of the following plasmids: Siglec1, TRIM27, and WT or mutant TBK1. An empty pcDNA3.1 vector was used to maintain equal amounts of DNA among wells. Cells were collected at $24 \mathrm{~h}$ after transfection, and luciferase activity was measured with a Dual-Luciferase Assay (Promega, Madison, WI, USA) with a Luminoskan Ascent Luminometer (Thermo Scientific, Hanover Park, IL, USA) as described previously [39]. Reporter gene activity was determined by normalizing Firefly luciferase activity to Renilla luciferase activity.

\section{Immunoblot and immunoprecipitation}

Cells were lysed using Cell Lysis Buffer (Cell Signaling Technology) supplemented with cocktail protease inhibitor (Calbiochem). Protein concentrations of the extracts were measured using a BCA assay (Pierce, Rockford, IL, USA) and equalized with the extraction reagent. Equivalent amounts of extracts were used for IP, or were loaded and subjected to SDS-PAGE, transferred onto nitrocellulose membranes, and then blotted as described previously [41]. For IP, whole-cell extracts were prepared after transfection or stimulation with appropriate ligands, followed by incubation overnight with the appropriate antibodies plus Protein A/G beads. Beads were washed five times and eluted as described previously [10].

\section{Statistical analysis}

All statistical analyses were performed by Student's $t$-test using Prism (version 5.0; GraphPad). Statistical values achieving $P<0.05$ were considered to be statistically significant.

\section{Acknowledgments}

We thank Ms Tingting Fang, Mei Jin, and Yan Li for technical assistance, Drs Chaofeng Han, Xingguang Liu, Sheng Xu, Yanmei Han, and Taoyong Chen for valuable discussions. This work was supported by the National Basic Research Program of China (2013CB530502 and 2012CB518900), and the National Natural Science Foundation of China (81123006, 31300718, 31370864, and 81422037).

\section{References}

1 Liu SY, Aliyari R, Chikere K, et al. Interferon-inducible cholesterol-25-hydroxylase broadly inhibits viral entry by pro- 
duction of 25-hydroxycholesterol. Immunity 2013; 38:92-105.

2 Ivashkiv LB, Donlin LT. Regulation of type I interferon responses. Nat Rev Immunol 2014; 14:36-49.

3 Schneider WM, Chevillotte MD, Rice CM. Interferon-stimulated genes: a complex web of host defenses. Annu Rev Immunol 2014; 32:513-545.

4 Hertzog PJ, Williams BR. Fine tuning type I interferon responses. Cytokine Growth Factor Rev 2013; 24:217-225.

5 O'Neill LA, Golenbock D, Bowie AG. The history of Tolllike receptors - redefining innate immunity. Nat Rev Immunol, 2013; 13:453-460.

6 Broz P, Monack DM. Newly described pattern recognition receptors team up against intracellular pathogens. Nat Rev Immunol 2013; 13:551-565.

7 Yoo JS, Kato H, Fujita T. Sensing viral invasion by RIG-I like receptors. Curr Opin Microbiol 2014; 20C:131-138.

8 Cui J, Zhu L, Xia X, et al. NLRC5 negatively regulates the NF-kappaB and type I interferon signaling pathways. Cell 2010; 141:483-496.

9 Cui J, Li Y, Zhu L, et al. NLRP4 negatively regulates type I interferon signaling by targeting the kinase TBK1 for degradation via the ubiquitin ligase DTX4. Nat Immunol 2012; 13:387-395.

10 Chen W, Han C, Xie B, et al. Induction of Siglec-G by RNA viruses inhibits the innate immune response by promoting RIG-I degradation. Cell 2013; 152:467-478.

11 Pillai S, Netravali IA, Cariappa A, Mattoo H. Siglecs and immune regulation. Annu Rev Immunol 2012; 30:357-392.

$12 \mathrm{Yu}$ X, Feizpour A, Ramirez NG, et al. Glycosphingolipid-functionalized nanoparticles recapitulate CD169-dependent HIV-1 uptake and trafficking in dendritic cells. Nat Commun 2014; 5:4136.

13 Delputte PL, Breedam VW, Delrue I, et al. Porcine arterivirus attachment to the macrophage-specific receptor sialoadhesin is dependent on the sialic acid-binding activity of the N-terminal immunoglobulin domain of sialoadhesin. J Virol 2007; 81:9546-9550.

14 Martinez-Pomares L, Gordon S. CD169 ${ }^{+}$macrophages at the crossroads of antigen presentation. Trends Immunol 2012; 33:66-70.

15 Kawasaki N, Vela JL, Nycholat CM, et al. Targeted delivery of lipid antigen to macrophages via the CD169/sialoadhesin endocytic pathway induces robust invariant natural killer T cell activation. Proc Natl Acad Sci USA 2013; 110:7826-7831.

16 Zhao W. Negative regulation of TBK1-mediated antiviral immunity. FEBS Lett 2013; 587:542-548.

17 Li S, Wang L, Berman M, Kong YY, Dorf ME. Mapping a dynamic innate immunity protein interaction network regulating type I interferon production. Immunity 2011; 35:426-440.

18 Ye JS, Kim N, Lee KJ, et al. Lysine 63-linked TANK-binding kinase 1 ubiquitination by mindbomb E3 ubiquitin protein ligase 2 is mediated by the mitochondrial antiviral signaling protein. J Virol 2014; 88:12765-12776.

19 Wang C, Chen T, Zhang J, et al. The E3 ubiquitin ligase Nrdp1 'preferentially' promotes TLR-mediated production of type I interferon. Nat Immunol 2009; 10:744-752.

20 Zhang M, Wang L, Zhao X, et al. TRAF-interacting protein (TRIP) negatively regulates IFN-beta production and antiviral response by promoting proteasomal degradation of
TANK-binding kinase 1. J Exp Med 2012; 209:1703-1711.

21 Chung HY, Gu M, Buehler E, MacDonald ER, Rice CM. Seed sequence-matched controls reveal limitations of small interfering RNA knockdown in functional and structural studies of hepatitis C virus NS5A-MOBKL1B interaction. J Virol 2014; 88:11022-11033.

22 Mori M, Yoneyama M, Ito T, et al. Identification of Ser-386 of interferon regulatory factor 3 as critical target for inducible phosphorylation that determines activation. $\mathrm{J}$ Biol Chem 2004; 279:9698-9702.

23 Blasius AL, Cella M, Maldonado J, Takai T, Colonna M. Siglec-H is an IPC-specific receptor that modulates type I IFN secretion through DAP12. Blood 2006; 107:2474-2476.

24 Taylor VC, Buckley CD, Douglas M, et al. The myeloid-specific sialic acid-binding receptor, $\mathrm{CD} 33$, associates with the protein-tyrosine phosphatases, SHP-1 and SHP-2. J Biol Chem 1999; 274:11505-11512.

25 An H, Zhao W, Hou J, et al. SHP-2 phosphatase negatively regulates the TRIF adaptor protein-dependent type I interferon and proinflammatory cytokine production. Immunity 2006; 25:919-928.

26 Radivojac P, Vacic V, Haynes C, et al. Identification, analysis, and prediction of protein ubiquitination sites. Proteins 2010; 78:365-380.

27 Crocker PR, Paulson JC, Varki A. Siglecs and their roles in the immune system. Nat Rev Immunol 2007; 7:255-266.

28 Crow MK, Olferiev M, Kirou KA. Targeting of type I interferon in systemic autoimmune diseases. Transl Res 2015; 165:296-305.

29 Zou Z, Chastain A, Moir S, et al. Siglecs facilitate HIV-1 infection of macrophages through adhesion with viral sialic acids. PLoS One 2011; 6:e24559.

30 Delputte PL, Van Gorp H, Favoreel HW, et al. Porcine sialoadhesin (CD169/Siglec-1) is an endocytic receptor that allows targeted delivery of toxins and antigens to macrophages. PLoS One 2011; 6:e16827.

31 Asano K, Nabeyama A, Miyake Y, et al. CD169-positive macrophages dominate antitumor immunity by crosspresenting dead cell-associated antigens. Immunity 2011; 34:85-95.

32 Versteeg GA, Rajsbaum R, Sanchez-Aparicio MT, et al. The E3-ligase TRIM family of proteins regulates signaling pathways triggered by innate immune pattern-recognition receptors. Immunity 2013; 38:384-398.

33 Gack MU, Shin YC, Joo CH, et al. TRIM25 RING-finger E3 ubiquitin ligase is essential for RIG-I-mediated antiviral activity. Nature 2007; 446:916-920.

34 Tsuchida T, Zou J, Saitoh T, et al. The ubiquitin ligase TRIM56 regulates innate immune responses to intracellular double-stranded DNA. Immunity 2010; 33:765-776.

35 Zhang Z, Bao M, Lu N, et al. The E3 ubiquitin ligase TRIM21 negatively regulates the innate immune response to intracellular double-stranded DNA. Nat Immunol 2013; 14:172-178.

36 Zurek B, Schoultz I, Neerincx A, et al. TRIM27 negatively regulates NOD2 by ubiquitination and proteasomal degradation. PLoS One 2012; 7:e41255.

37 Neel BG, Gu H, Pao L. The 'Shp'ing news: SH2 domain-containing tyrosine phosphatases in cell signaling. Trends Biochem Sci 2003; 28:284-293. 
38 Chen T, Yang M, Yu Z, et al. Small GTPase RBJ mediates nuclear entrapment of MEK1/MEK2 in tumor progression. Cancer Cell 2014; 25:682-696.

39 Hou J, Zhou Y, Zheng Y, et al. Hepatic RIG-I predicts survival and interferon-alpha therapeutic response in hepatocellular carcinoma. Cancer Cell 2014; 25:49-63.

40 Wang P, Xue Y, Han Y, et al. The STAT3-binding long non- coding RNA lnc-DC controls human dendritic cell differentiation. Science 2014; 344:310-313.

41 Liu J, Han C, Xie B, et al. Rhbdd3 controls autoimmunity by suppressing the production of IL-6 by dendritic cells via K27linked ubiquitination of the regulator NEMO. Nat Immunol 2014; 15:612-622.

(Supplementary information is linked to the online version of the paper on the Cell Research website.) 